\title{
Kane Tanaka's 119 birthday and the Supercentenarians' age estimation. Further remarks on the oldest old record of 122 years by Jeanne Calment
}

\author{
Christos H Skiadas
}

Christos H Skiadas, PhD

Former Vice Rector, Technical University of Crete, Chania, Crete, Greece

Chair: ISAST International, Athens, Greece

Email: skiadas@cmsim.net

Web: www.cmsim.net

\begin{abstract}
In a previous study, based on the large number of centenarians in Japan, we had constructed a model to estimate the supercentenarians in the country, https://doi.org/10.1007/978-3-319-76002-5_2 . The model was published in Volume 46 of The Springer Series on Demographic Methods and Population Analysis. Projections are done and forecasts are made for the maximum expected age at death of record holder Kane Tanaka, now at 119 years old. Now, February 22, 2022, we have used the Saito-Ishii-Robine (2021) death data set for females (100 to 118 years old) in Japan from 1951 to 2015 to test an advanced model. We fit this model to data from 100 to 109 years of age and make projections from 110 to 119 years of age. The fit and projections apply perfectly. We have also used data from the Human Mortality Database (HMD) for female deaths ( 100 to +110 years old) in Japan from 1950 to 2019 . The fit and projections verify the expected one supercentenarian at 119 years of age. The same model was applied to centenarian female deaths from 1950 to 2019 in a large number of countries (Europe-USA-Canada-Australia-New Zealand and Japan). By this method, a large amount of death data is selected, adequate to find extreme age supercentenarians. The fit and projections led to at least one supercentenarian at 122 years of age; that is Jeanne Calment's record. Having estimated the two parameters of the model (slope and curvature) for these countries, we have fitted the Gerontology Research Group (GRG) database set, which led to an expectation of a supercentenarian at 120 years of age and a good probability for a 121-year-old supercentenarian in the world. A recent publication by Lenart A., Aburto J.M., Stockmarr A., Vaupel J.W. (2021) confirms our findings from 2018 regarding Calment's record. Our provided formula estimates one supercentenarian at age 122 in the year 2045 (Skiadas, 2018) to reach the Calment's record.
\end{abstract}

Keywords: Centenarians, Supercentenarians, Maximum Reported Age at Death (MRAD), MRAD formula

\section{Introduction}

A few years ago, following a Guinness Records announcement, Kane Tanaka from Fukuoka, Japan, was officially confirmed as the oldest person living at 116 years, 66 days old, as of 9 March 2019.

Created, February 22, 2022 
However, our study from 2017 predicted for 2020 at least one supercentenarian in Japan, aged 2018 years. That is why Kane Tanaka's $117^{\text {th }}$ birthday on Thursday January 2, 2020 was a critical point for our proposed theory and the mathematical expression providing the highest expected age of a living person in Japan.

A simple equation for the Maximum Reported Age at Death (MRAD=0.1774*YEAR-240.53) for Japanese women provides estimates for the MRAD predicted. According to these estimates, the expected MRAD for 2020 is 117.8 years of age. Accordingly, a MRAD at 122.45 years of age should be reached by 2046.11 years and a MRAD at 125 years of age is expected by 2060 .

The findings of our study on Remarks on "Limits to Human Lifespan" (Skiadas, 2018) were published in The Springer Series on Demographic Methods and Population Analysis 46, whereas a related study was published in the previous Volume 45 of the same Springer Series (Skiadas \& Skiadas, 2018).

Several technical details were solved, especially by transforming the death data sets with the appropriate logarithmic method and finding the last surviving person or the related MRAD.

In a recent publication, Lenart A., Aburto J.M., Stockmarr A., Vaupel J.W. (2021) on "The Human Longevity Record May Hold for Decades: Jeanne Calment's Extraordinary Record Is Not Evidence for an Upper Limit to Human Lifespan" verify our findings. They verify the expected time to break the Calment's record to be about the mid-year 2045 very close to our estimates from 2017 to be at 2046.11 years. See below their paragraph on this matter and compare with our figure 1 below reproducing the related figure from 2018 paper.

Calment's record is likely to be broken when the probability of someone living longer than 122.45 exceeds $50 \%$. To reach this level, we need to mix the estimated number of people attaining age 110 in the period prior to the end of 2010 (165 people) with 3713 new supercentenarians. The required number of new supercentenarians would not be reached until near the end of 2033. Then it would take an additional 12.45 years for one of them to break Calment's record, so about mid-year 2045 , assuming the annual probability of death after age 110 remains at 0.5 .

Japanese data for centenarians and supercentenarians were ideal for our study. According to the data collected from the Human Mortality Database for 2018, the persons of 100 years old and more exceeded 70000 with 62572 women and only 8661 men. The women of 110 years and higher, called supercentenarians, are 108 and the expected number of supercentenarians is only 1 per 580 or approximately 2 per 1000. This is double than in Europe. Evenmore, as the trend is steadily growing, the estimates can be satisfactory.

Also recently Pearce and Raftery (2021) in their study, estimate that is likely to expect supercentenarians at ages 122, 126, 128 and 130 this century with probabilities $100 \%, 89 \%, 44 \%$ and $13 \%$, respectively something that we have already estimated for Japan (see figure 1 and the related equation for MRAD). 


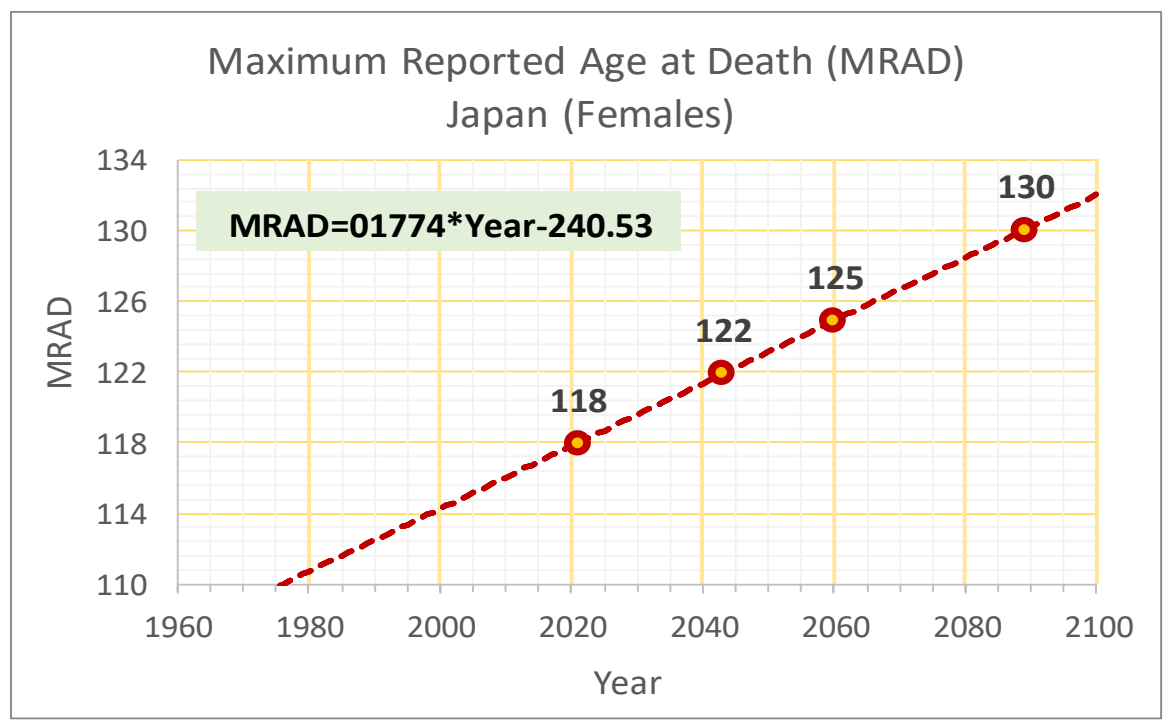

Fig.1. Fit and Projections for MRAD in Japan females (Reproduced from Skiadas, 2018, https://doi.org/10.1007/978-3-319-76002-5_2 )

The Japan female population at 100 years of age steadily increases from 1950 and onwards. Following TOMOE ISHIKAWA of Asahi Shimbun on September 14, 2021, Japan's number of centenarians is set to hit a high of 86.510 , including 76.450 women and only 10.060 men.

This is very important, indicating an increase in the number of centenarians and supercentenarians (aged $100+$ years of age) and thus increasing the probability of finding MRAD at higher ages.

Japanese women have a very high life expectancy at birth, ranked in one of the top few places in the World. More important is that $0.1 \%$ of the women population in 2018 is 100 years old or higher, forming the pool for the future supercentenarians.

The example of Japan studied, strongly supports a growth of the maximum expected life span at least in some countries. And this is before the changes expected to come due to advances in science and medicine. Clearly, centenarians and supercentenarians have spent a large part of their lives in the old system of medical science and services.

\section{The Mathematical Model}

Following the theory proposed in Skiadas (2018), and Skiadas \& Skiadas $(2018,2020)$, we approach the fast declining form of the number of deaths at very high ages and especially those of the centenarians and supercentenarians in a population by an equation of the form:

$$
\log (D(x))=a-(b x)^{c} \text { or } D(x)=\exp \left(a-(b x)^{c}\right)
$$

Where $D(x)$ represent the number of deaths, $a, b$ and $c$ are parameters and $x$ is the age or time. The latter is denoted by $t$.

The MRAD is estimated when $\log (D(x))=a-(b x)^{c}=0$, thus it is at MRAD $=\sqrt[c]{a} / b=a^{\left(\frac{1}{c}\right)} / b$

Note that for $c=1$ the simpler form results. That is:

With $M R A D=a / b$

$$
\log (D(x))=a-b x
$$


The logarithm of the centenarian and supercentenarian data provides a decreasing pattern with a negative curvature that is represented by the curvature parameter $c$. The model with zero curvature $(c=1)$ usually overestimates the future trends.

In the case of centenarians and supercentenarians the age starts at $x=100$ years of age. Accordingly, the equation fitted to data takes the form:

$$
\log (D(x))=a-(b(x-100))^{c}
$$

The formula for MRAD is $\quad \operatorname{MRAD}=100+\sqrt[c]{a} / b=100+a^{\left(\frac{1}{c}\right)} / b$

\section{The Saito-Ishii-Robine (2021) centenarian and supercentenarian death data in Japan (1951 to 2015)}

The death data sets from the Human Mortality Database provide yearly data from 100 to 109 years of age and then, for +110 years, that is for the supercentenarians, only a single number as a summation is provided.

However, to test the proposed model on how well it fits the data including the detailed analysis of all death data set, including from 100 years until the last one a valuable data set is needed. This very important information, including death data from 1951 to2015 is provided by Saito-Ishii-Robine (2021) in their paper (see Table I). Details for Japan centenarians and supercentenarians can also be found in Saito (2010).

We fit the model (1) to female data from 100 to 109 years of age and make projections for 2018. The Root Mean Squared Error is RMSE $=0.01343$ for the fit and $\mathrm{RMSE}=0.2189$ for the projections. See figure 2 for the fit and projections on the logarithm of deaths and figure 3 for the number of deaths. The estimated parameters are $a=4.8658, b=0.1987$, and $c=1.2680$ and the MRAD $=117.52$ years from (2).

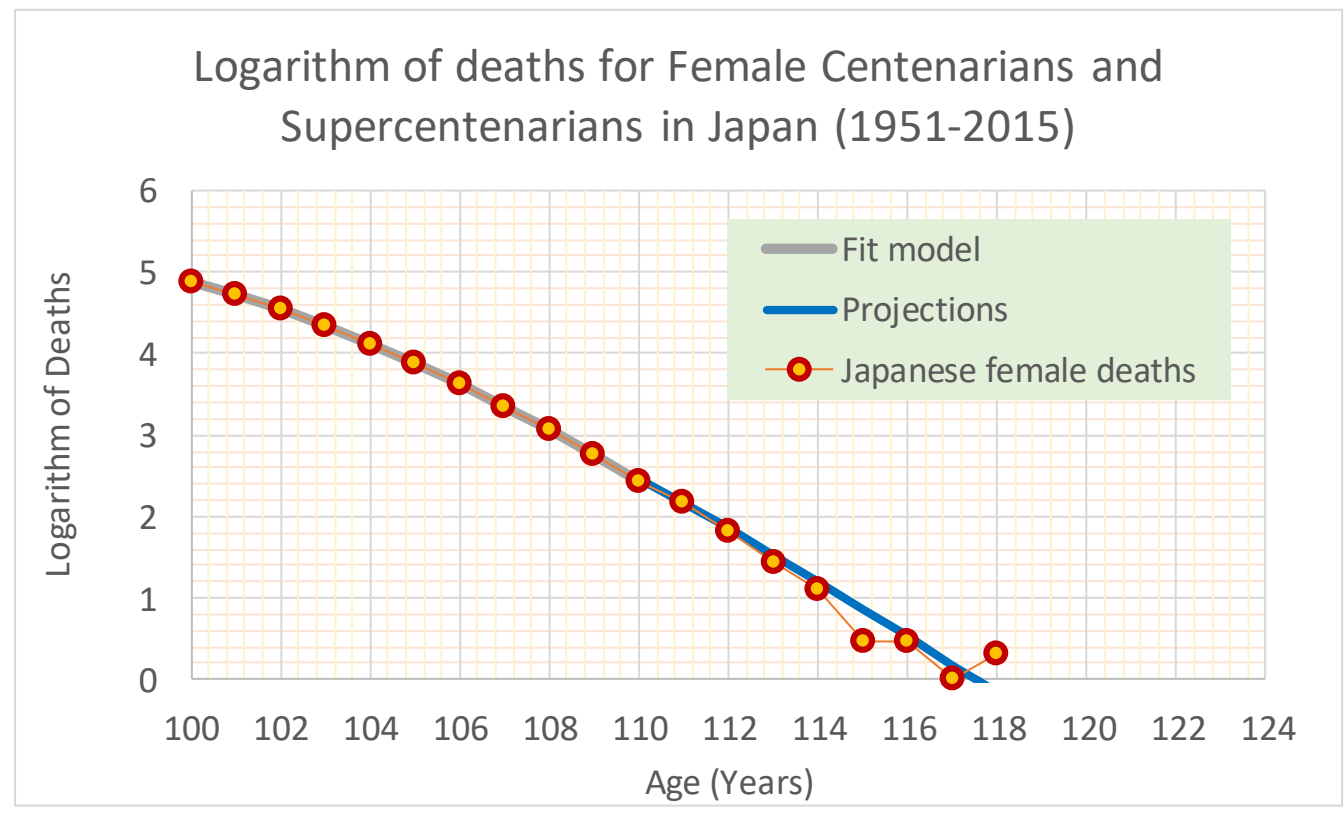

Fig. 2. Fit and projections to Japanese female data from 1951 to 2015 in logarithmic form. The red bullets represent the Centenarians and Supercentenarians deaths from the Saito-Ishii-Robine list. 


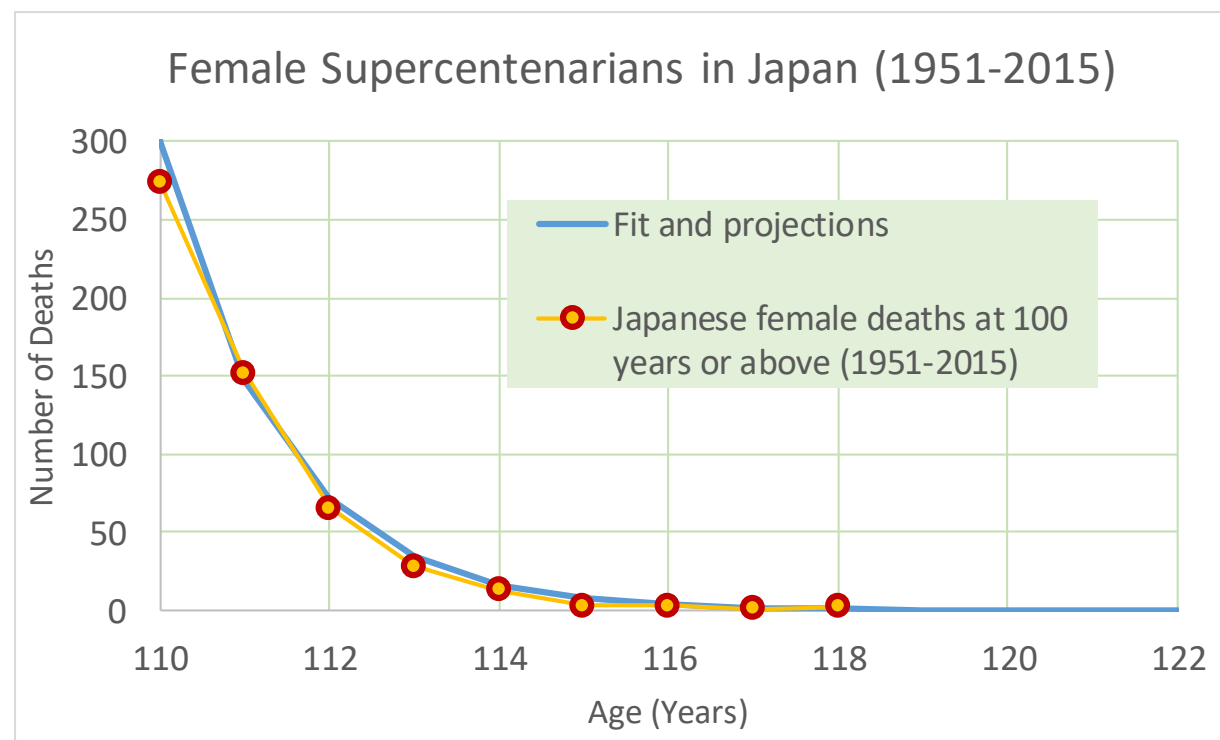

Fig. 3. Fit and projections to Japanese female data from 1951 to 2015. The red bullets represent the Centenarians and Supercentenarians deaths from the Saito-Ishii-Robine list (Table I).

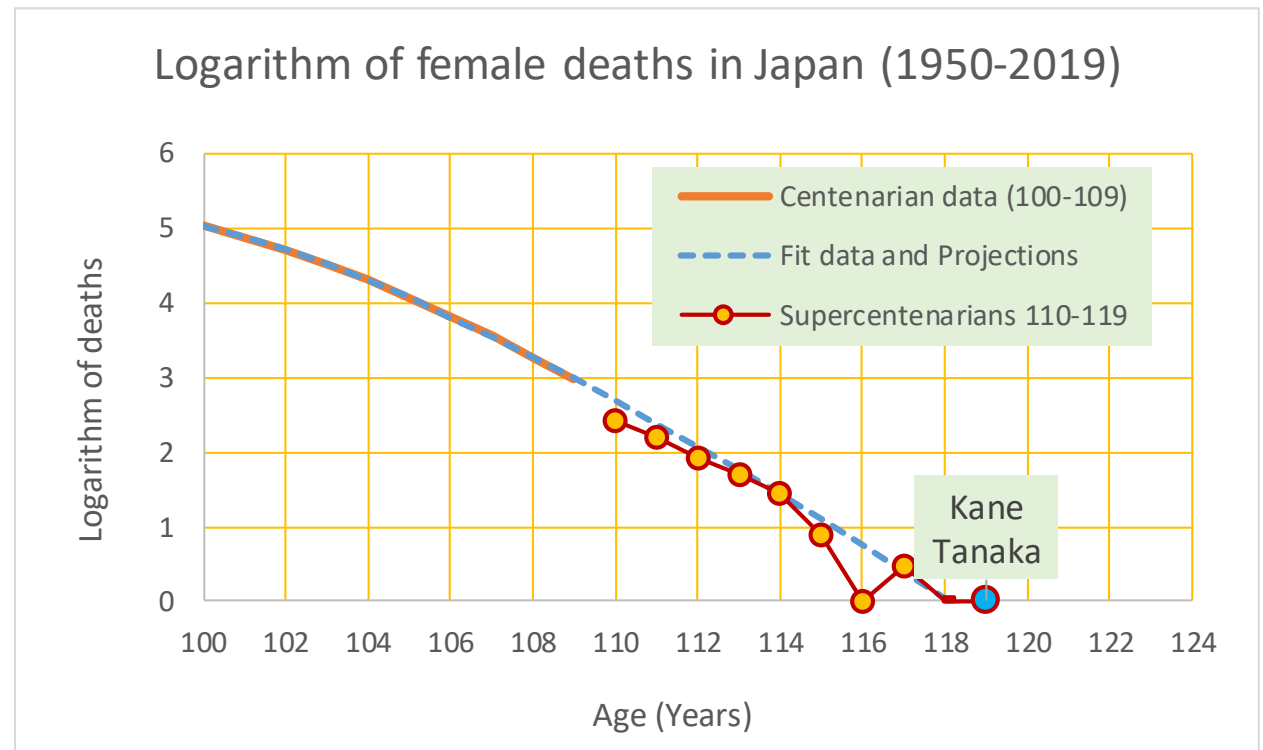

Fig. 4. Fit and projections to Japanese female data from 1950 to 2019 in logarithmic form. The red bullets represent the supercentenarians from the GRG list for the same period.

The formula (1) is applied to the logarithm of the Japanese female death data sets for the period 19502019 provided by the Human Mortality Database (HMD). The formula is fitted to the centenarian data from 100 to 109 years of age by using the Excel Solver facility of the related Excel program. The program is provided upon request. The fit data and projections are illustrated by the blue dashed line in figure 4. The logarithm of the number of supercentenarians is presented by the red bullets.

The number of centenarians and supercentenarians computed and provided by the GRG database is illustrated in figure 5. The estimated number is larger than that of the GRG database. Clearly, a number 
of supercentenarians is difficult to be found and validated. However, the missing number is not very large. Evenmore, the estimated end age or maximum reported age at death (MRAD) is at 118 years of age as it was also provided in our previous study from 2017, expecting 118.17 years of age in 2022. Our estimates today, February 22, 2022, provide at least 1.134 persons for 118 and 0.4978 persons for 119 years, that corresponds to Kane Tanaka (see figures 4 and 5).

Looking at Kane Tanaka's case, reaching 119 years of age and expecting to pass Sarah Knauss this April 2022 to be the $2^{\text {nd }}$ older person from the $3^{\text {rd }}$ place she holds today. By having 2 people reaching the 119 years of age and 3 who had reached the 117 years, and Lucile Randon from France, who celebrated her $118^{\text {th }}$ birthday on February 11,2022 , we are more optimistic about facing the 120 years ceiling earlier than 2032 as of our previous studies. However, the 122-year World record of Jeanne Calment is not far away from reasonable approach in the future.

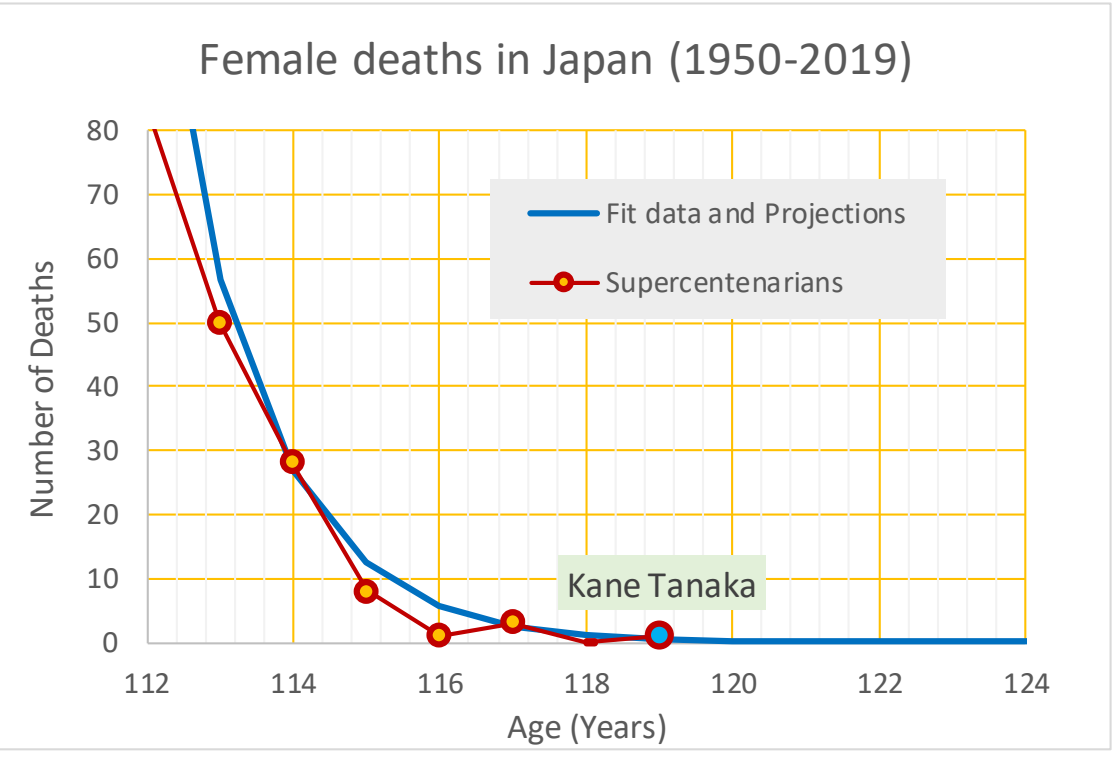

Fig. 5. Number of Supercentenarians in Japan. Fit and projections and GRG data

\section{Validating the MRAD}

\section{The Data. Fit and Projections}

It is well known that the number of centenarians and supercentenarians increases according to the number of deaths or to the population.

However, the simpler is to search for centenarians and supercentenarians into the number of deaths in a long time horizon than into the living population. The after the second World War period, starting from 1950-51 until today is important for our studies. It is a relatively calm period with fewer war activities and more social organization. Countries are developing and applying demographic issues resulting in keeping records and collecting data in the majority of them. Centenarian and supercentenarian data sets can be found, analyzed and verified.

Improvements in the way of living, in medicine, health treatments have turned to increasing the number of over 100 people rapidly, with the number of female centenarians to be larger than males and the same holds for supercentenarians as well (over 110 years of age). Soon after the systematic work of researchers, 
it was possible to "validate" the correct age of supercentenarians by referring to the maximum reported age at death (MRAD). Jeanne Calment from France died at 122 years and 164 days of age in 1997 and Sarah Knauss at 119 years and 97 days of age in 1999 in the USA. Both cases were considered as exceptional cases of long-living persons, with Jeanne Calment characterized as highly exceptional, leading to debates. However, starting from Sarah Knauss' case with the 119 years' record, it was also thought that it was an exceptional case as well. That is why Kane Tanaka reaching the 117-year record as the oldest verified living person in the world was celebrated by Guinness World Record holder. Two years later, in 2022, Kane Tanaka celebrated her new 119 years' world record on January 2, 2022, while she only needs another two months to be in second place in the world, passing Sarah Knauss' record. Evenmore, Lucile Randon from France celebrated 118 years of age on February 11, 2022, setting a new candidate for an exceptional high age supercentenarian. Following our study from 2017, it was estimated a supercentenarian in Japan at 118 years of age in 2020 that was reached by Kane Tanaka in the previous year. It remains the very exceptional case of Jeanne Calment. That of another woman from France, Lucile Randon, reaching 118 years of age is very encouraging for verifying for exceptional high age cases in France. It was also detected in our study published in 2018 (Remarks on "Limits to Human Lifespan", Skiadas 2018).

Collecting the data for such an exceptional case, the total female deaths from 1950 to 2019 from the Human Mortality Database are selected. Note that for some cases the collected data starts later than 1950 or ends before 2019. The total deaths include Europe, including the UK, the USA and Canada, Australia and New Zealand, and Japan. See the provided Table II where the centenarians at 100 years of age are 723700 and the total supercentenarians at $110+$ are 8920 . Data from the majority of countries in Africa, Central and South America, and Asia are not included in HMD.

We fit the logarithm of data by applying the model (1) proposed earlier. The estimated parameters are as follows: $b=0.19794, c=1.19507$ and $a=5.8477$ with Root Mean Square Error RMSE $=0.007235$. The zero logarithm case corresponds to MRAD=122.1449 years of age or at 122 years and 53 days. It is thus indicated that an exceptional age supercentenarian as Jeanne Calment was possible to appear in the period 1950 to 2019. Note that the calculations done found the curvature of the logarithm, which is very important for the estimates. In the following, the curvature that is expressed by parameter $c$ and the form expressed by parameter $b$ are supposed to remain stable while parameter $a$ related to the number of deaths is changing. Accordingly, fitting the parameter $a$ to GRG data from the February 20, 2022, we receive $a=5.3731$ corresponding to 120.637 years of age, which is 120 years and 233 days. Note that this is an estimate for one supercentenarian at this age, which is at zero logarithm. Our estimates for $\log (D(x))=-0.11318$ provides an age of 121 years. At 122 years of age $\log (D(x))=-0.4599$, corresponding to 0.3468 or $34.68 \%$. See figure 6 for fit and projections in logarithmic form and figure 7 for the number of deaths and Table III for the estimated parameters of the model.

Finally, the estimates from HMD data sets suggest at least 122 years of age in the nowadays data expecting higher estimates when data from China, India, and Brazil comes across along with data from other countries. More important are our estimates for fitting the model to recent GRG data (February 20, 2022) providing estimates for one supercentenarian at 120 years, expected something that Kane Tanaka could achieve next year or Lucile Randon in two years from now. Even more, the probability of expecting a supercentenarian at 121 years of age is also within our estimated future trends. 
It remains to check the periods when the extra high supercentenarians are expected to appear. Clearly the 1990-1999 decade was one of the best. However, the new decade starting at 2020 and continuing at 2022 with one living person at 119 and a second at 118 is promising for new records. It follows Tekla Juniewicz from Poland at 115 years of age followed by 12 persons of 114 years old to continue with new exceptional supercentenarian candidates the coming years.

\begin{tabular}{|l|l|l|l|l|l|}
\hline \multicolumn{6}{|l|}{ TABLE I. Centenarians and Supercentenarians Deaths in Japan (1951-2015) from Saito-Ishii-Robine } \\
\hline 100 & 77075 & 107 & 2290 & 114 & 13 \\
\hline 101 & 52752 & 108 & 1138 & 115 & 3 \\
\hline 102 & 34456 & 109 & 582 & 116 & 3 \\
\hline 103 & 21790 & 110 & 274 & 117 & 1 \\
\hline 104 & 13216 & 111 & 152 & 118 & 2 \\
\hline 105 & 7589 & 112 & 65 & & \\
\hline 106 & 4296 & 113 & 28 & & \\
\hline
\end{tabular}

\begin{tabular}{|l|l|l|l|l|l|}
\hline \multicolumn{5}{|l|}{ TABLE II. Centenarian and Supercentenarian Death Data (1950-2019) in Selected World Countries } \\
\hline Age (Year) & EU & Japan & USA+CA+AU+NZ & Total Sum & Log of Sum \\
\hline 100 & 360458.95 & 107510.23 & 255731.51 & 723700.69 & 5.859558987 \\
\hline 101 & 238840.31 & 75427.93 & 176715.13 & 490983.37 & 5.691066782 \\
\hline 102 & 153805.17 & 50272.03 & 118123.76 & 322200.96 & 5.50812683 \\
\hline 103 & 95359.86 & 32448.23 & 75988.10 & 203796.19 & 5.309196061 \\
\hline 104 & 56983.79 & 20177.78 & 47973.24 & 125134.81 & 5.097378139 \\
\hline 105 & 33103.16 & 11702.12 & 29167.34 & 73972.62 & 4.869071001 \\
\hline 106 & 18338.75 & 6644.96 & 16831.77 & 41815.48 & 4.621337086 \\
\hline 107 & 10231.97 & 3624.56 & 9671.25 & 23527.78 & 4.371580951 \\
\hline 108 & 5579.55 & 1812.77 & 5639.02 & 13031.34 & 4.114989076 \\
\hline 109 & 3071.65 & 947.43 & 3052.88 & 7071.96 & 3.849539796 \\
\hline $110+$ & 3428.07 & 860.28 & 4631.72 & 8920.07 & 3.950368263 \\
\hline
\end{tabular}

Total EU [Austria (1950-2019), Belarus (1960-2018), Belgium (1950-2019), Bulgaria (1950-2017), Croatia (2001-2019), Czechia (1950-2019), Denmark (1950-2019), Estonia (1960-2019), Finland (1950-2019), France (1950-2018), Germany (1990-2017), Greece (1981-2019), Hungary (1950-2019), Iceland (1950-2018), Ireland (1950-2017), Italy (1950-2018), Latvia (1960-2019), Lithuania (1960-2019), Luxembourg (1960-2019), Netherlands (1950-2019), Norway (1950-2019), Poland (1958-2019), Portugal (1950-2019), Russia (1960-2014), Slovakia (1950-2019), Slovenia (1983-2019), Spain (1950-2018), Sweden (1950-2019), Switzerland (1950-2019), UK (1950-2018), Ukraine (1960-2013).]

\begin{tabular}{|l|l|l|l|l|}
\hline \multicolumn{5}{|c|}{ TABLE III. Fit Model Parameters and Root Mean Square Error for World Countries and Japan } \\
\hline & b & c & a & RMSE \\
\hline Total Sum (World) & 0.1979 & 1.1951 & 5.8478 & 0.007235 \\
\hline Japan (Saito et al) & 0.1987 & 1.2680 & 4.8658 & 0.01343 \\
\hline Japan (1950-2019) & 0.1921 & 1.2900 & 5.0109 & 0.01292 \\
\hline
\end{tabular}




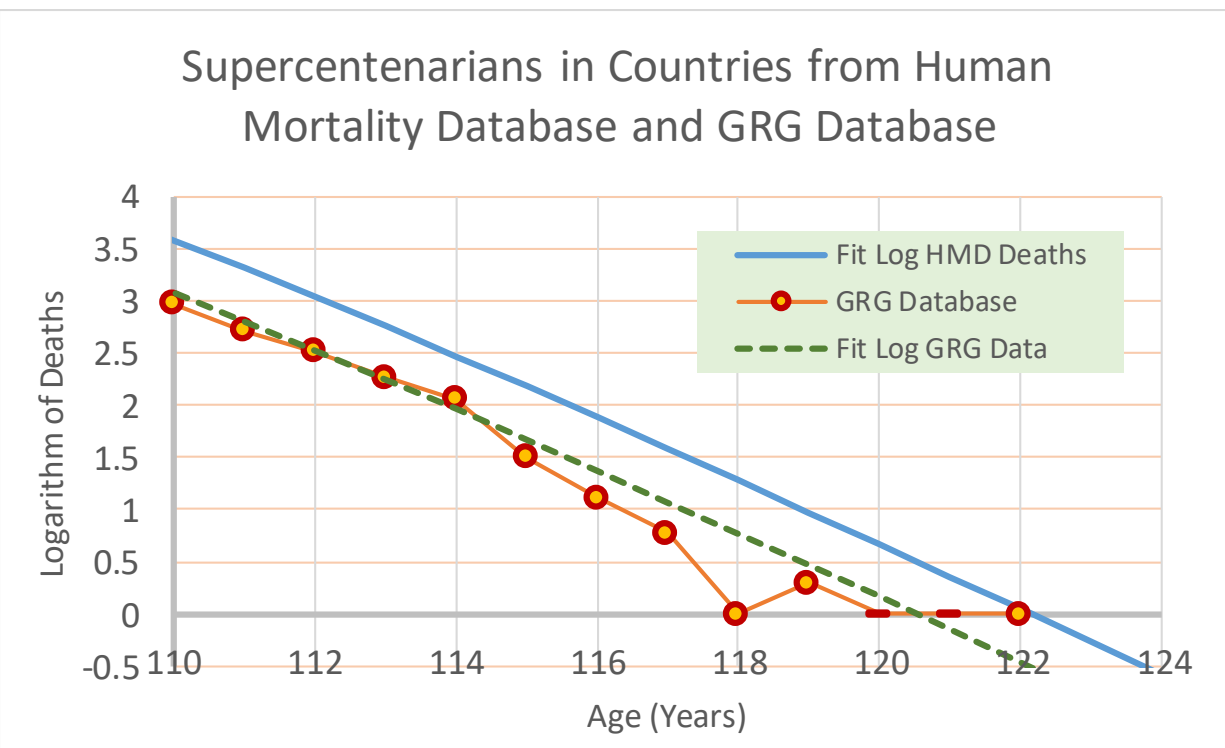

Fig. 6. Logarithm of Supercentenarians Deaths from Selected World Countries from the HMD

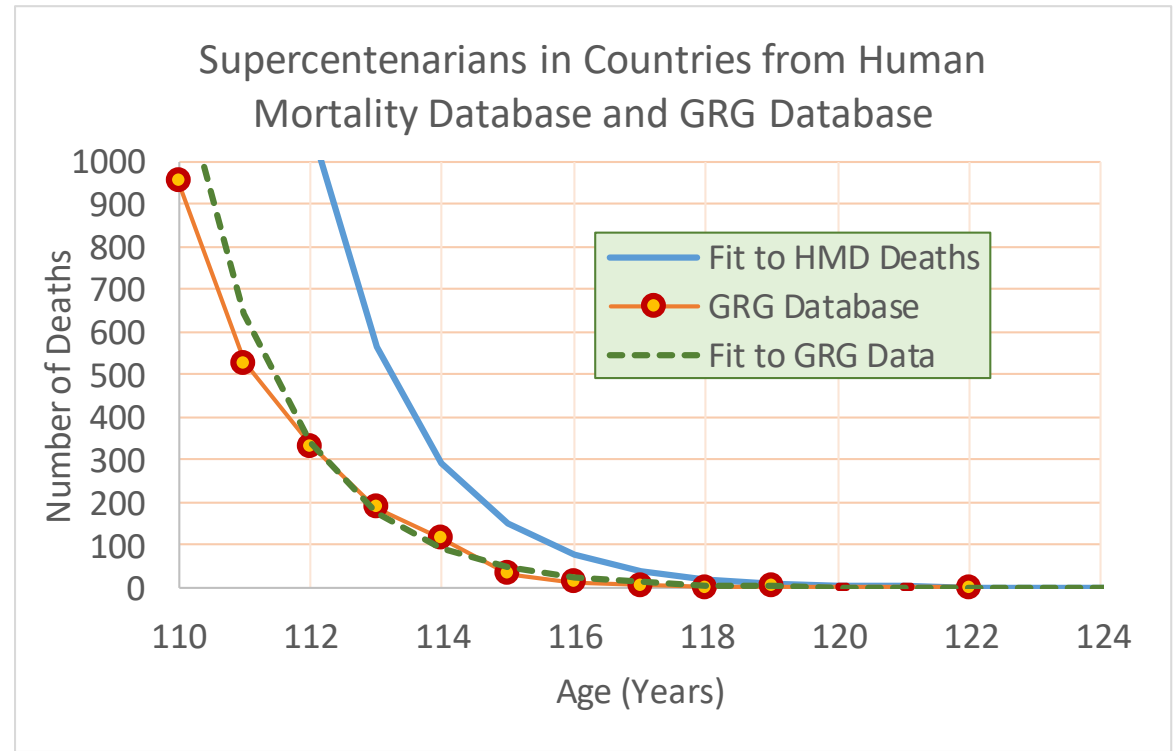

Fig. 7. Number of Supercentenarians from Selected World Countries from the HMD

\section{Summary and Conclusions}

We have developed a model and a methodology to study the centenarian and supercentenarian development in various countries and especially in Japan and a collection of World countries from HMD. Several important questions should be answered over the years. Debates came in previous years due to the lack of data, the systematic collection and the overestimation of our ability to solve problems. However, as far as the number of centenarians increases worldwide, the estimation or forecastspredictions of future supercentenarians is better. It is also straightened by the continuously enlarged number of supercentenarians over the years. The nowadays estimates by quantitative methods tend to support the expectation to bypass the oldest old record of 122.45 years by Jeanne Calment in the coming 
decades. To this end, we acknowledge the valuable support of the Japanese data provided in the paper by Saito-Ishii-Robine (2021) and the HMD data used in this paper as well.

*Announcements and further discussion on "Kane Tanaka 119 birthday and the Japan Supercentenarians age estimation" will be done in the Demographics2022 Workshop (7-10 June 2022, Athens, Greece, http://www.smtda.net/demographics2022.html ).

\section{References}

Lenart A., Aburto J.M., Stockmarr A., Vaupel J.W. (2021) The Human Longevity Record May Hold for Decades: Jeanne Calment's Extraordinary Record Is Not Evidence for an Upper Limit to Human Lifespan. In: Maier H., Jeune B., Vaupel J.W. (eds) Exceptional Lifespans. Demographic Research Monographs (A Series of the Max Planck Institute for Demographic Research). Springer, Cham. https://doi.org/10.1007/978-3-030-49970-9 5

Pearce, M., Raftery, A.E. (2021) Probabilistic forecasting of maximum human lifespan by 2100 using Bayesian population projections. Demographic Research: Volume 44, Article 52, 1271-1294 http://www.demographicresearch.org/Volumes/Vol44/52/

Saito, Y. (2010). Supercentenarians in Japan in H. Maier et al. (eds.), Supercentenarians, Demographic Research Monographs, DOI 10.1007/978-3-642-11520-2_5, (C) Springer-Verlag Berlin Heidelberg.

Saito Y., Ishii F., Robine JM. (2021) Centenarians and Supercentenarians in Japan. In: Maier H., Jeune B., Vaupel J.W. (eds) Exceptional Lifespans. Demographic Research Monographs (A Series of the Max Planck Institute for Demographic Research). Springer, Cham. https://doi.org/10.1007/978-3-030-49970-9 10

Skiadas C.H., Skiadas C. (2018) Remarks and Findings on "Evidence for a Limit to Human Life Span". In: Exploring the Health State of a Population by Dynamic Modeling Methods. The Springer Series on Demographic Methods and Population Analysis, vol 45. Springer, Cham. https://doi.org/10.1007/978-3-319-65142-2 7

Skiadas, C.H. (2018). Remarks on "Limits to Human Lifespan", in Demography and Health Issues: Population Aging, Mortality and Data Analysis. The Springer Series on Demographic Methods and Population Analysis 46. Springer, Chum, Switzerland. https://doi.org/10.1007/978-3-319-76002-5, https://link.springer.com/chapter/10.1007/978-3$\underline{319-76002-5 \quad 2}$

Skiadas C.H., Skiadas C. (2020) How the unsolved problem of finding the Healthy Life Expectancy (HLE) in the far past was resolved: The case of Sweden (1751-2016) with forecasts to 2060 and comparisons with HALE. SocArXiv Papers, https://osf.io/preprints/socarxiv/akf8v/ 